\title{
Correction to: Effects of Moisture-Induced Crystallization on the Aerosol Performance of Spray Dried Amorphous Ciprofloxacin Powder Formulations
}

Nivedita Shetty' • Lingfei Zeng' - Sharad Mangal' • Haichen Nie ${ }^{2}$ - Matthew R. Rowles ${ }^{3}$ Rui Guo' • Youngwoo Han ' Joon Hyeong Park ${ }^{4}$ Qi (Tony) Zhou'

Published online: 8 February 2018

(C) Springer Science+Business Media, LLC, part of Springer Nature 2018

\section{Correction to: Pharm Res}

\section{https://doi.org/I0.1007/s I 1095-0 I7-228 I-5}

Page 4, right column, under section heading "In-Vitro Aerosol Performance", line 11:

" $<4 \mathrm{kPa}$ (approximately $3.2 \mathrm{kPa}$ at $100 \mathrm{~L} / \mathrm{min}$ )" should be changed to "approximately $4 \mathrm{kPa}$ at $100 \mathrm{~L} / \mathrm{min}$ ".

The online version of the original article can be found at https://doi.org/l 0 . |007/s | |095-0 |7-228|-5

\footnotetext{
Qi (Tony) Zhou

tonyzhou@purdue.edu
}

Department of Industrial and Physical Pharmacy, College of Pharmacy

Purdue University, 575 Stadium Mall Drive

West Lafayette, Indiana 47907, USA

2 Teva Pharmaceuticals, I 45 Brandywine Pkwy

West Chester, Pennsylvania 19380, USA

3 Department of Physics and Astronomy, Curtin University Bentley, WA 6102, Australia

4 Birck Nanotechnology Center, Purdue University

I 205 West State Street, West Lafayette, Indiana 47907, USA 